\title{
Alterações bucais associadas à presença de atopia em crianças: um estudo transversal
}

\author{
Oral changes associated with the presence of atopy in children: a cross- \\ sectional study \\ Bruna Mara RUAS ${ }^{a}\left(\mathbb{D}\right.$, , Marianne de Oliveira NASCIMENTO $\left.^{b}{ }^{(}\right)$, Marceli DE FRANÇA ${ }^{c}(\mathbb{D}$,

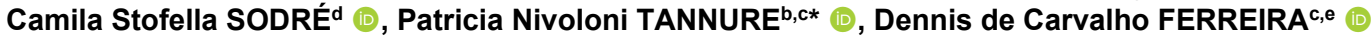 \\ aUSP - Universidade de São Paulo, Bauru, SP, Brasil

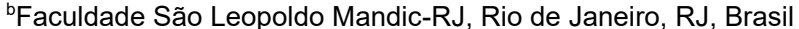 \\ 'UVA - Universidade Veiga de Almeida, Rio de Janeiro, RJ, Brasil \\ dUFRJ - Universidade Federal do Rio de Janeiro, Departamento de Clínica Médica, Rio de Janeiro, RJ, Brasil \\ eUniversidade Estácio de Sá, Rio de Janeiro, RJ, Brasil
}

\begin{abstract}
Como citar: Ruas BM, Nascimento MO, De França M, Sodré CS, Tannure PN, Ferreira DC. Alterações bucais associadas à presença de atopia em crianças: um estudo transversal. Rev Odontol UNESP. 2020;49:e20200067.

https://doi.org/10.1590/1807-2577.06720
\end{abstract}

\begin{abstract}
Resumo
Introdução: É por meio da interação entre ambiente, indivíduo, sua predisposição à atopia e exposição a alérgenos que surgem as doenças alérgicas. Em crianças atópicas, foi observada uma alta prevalência de padrão respirador bucal, assim como resposta positiva a testes alérgicos. Essas alterações respiratórias possuem uma associação com deformidades orofaciais, especialmente as maloclusões. Objetivo: Identificar a frequência de crianças e adolescentes portadores de alergias e buscar uma provável associação entre atopia e maloclusão, traumatismos dentoalveolares e hábitos bucais viciosos. Material e método: Foram avaliados prontuários de crianças e adolescentes atendidos em duas instituições particulares de ensino da cidade do Rio de Janeiro no período compreendido entre agosto de 2017 e julho de 2018. Foram coletados dados do prontuário relacionados ao histórico médico, odontológico e de alergias. Os dados foram analisados descritivamente e por meio do teste qui-quadrado $(\mathrm{p}<0,05)$. Resultado: Um total de 136 prontuários foi considerado elegível para avaliação. Durante a anamnese, 49 responsáveis (36\%) relataram histórico de alergias em suas crianças e adolescentes. A prevalência de hábitos bucais viciosos foi verificada em 87 pacientes (64\%), sendo presente em 65,3\% de crianças e adolescentes que apresentavam atopia $(p<0,05)$. Conclusão: A prevalência de alergias relatada nesta população foi de 34,6\%, e foi observada ainda uma alta prevalência de hábitos bucais viciosos e traumatismos dentoalveolares nesses pacientes. Foram constatadas associações significativas entre presença de atopias e histórico de traumatismos dentários, maloclusão e hábitos viciosos, demonstrando haver correlação entre atopia e alguns aspectos de saúde oral.
\end{abstract}

Descritores: Alergia; maloclusão; rinite alérgica; asma; boca.

\begin{abstract}
Introduction: Allergic diseases appear through the interaction between the environment, the individual, their predisposition to atopy and exposure to allergens. In atopic children there is a high prevalence of mouth breathers, as well as positive response to allergic prick tests. These respiratory changes have an association with orofacial deformities especially malocclusions. Objective: To identify the frequency of children and adolescents that have atopies and search for a probable association between atopy and oral aspects like malocclusion and vicious oral habits. Material and method: Medical records were taken of children and adolescents seen at two educational institutions in the city of Rio de Janeiro in the period between August 2017 and July 2018. Data were collected from medical records related to medical history, history of atopies and dental history. The data were transmitted descriptively through the chi-square test $(p<0.05)$. Result: A total of 136 records were considered eligible for evaluation. During the anamnesis, $49(36.0 \%)$ parents reported a history of atopy in their children adolescents. The prevalence of vicious oral habits was found in 87 children and adolescents (64.0\%), being presented in $65.3 \%$ of the patients who had atopy $(p<0.05)$. Conclusion: It can be observed that the prevalence of atopy reported in this population was $34.6 \%$ and there was also a high prevalence of vicious oral habits and dento-alveolar trauma in these patients. Relevant associations between the presence of atopies and history of dental trauma, malocclusion and the presence of oral vicious habits were observed, demonstrating a correlation between atopy and some aspects of oral health.
\end{abstract}

Descriptors: Allergy; malocclusion; allergic rhinitis; asthma; mouth. 


\section{INTRODUÇÃO}

A atopia caracteriza-se como uma disfunção do sistema imunológico, que pode manifestar-se individualmente ou em associação com imunodeficiências ou demais estados das doenças autoimunes. As doenças alérgicas (rinite alérgica, asma, eczema atópico, urticária, entre outras) surgem pela interação entre uma tendência atópica e o ambiente (que expõe o indivíduo a múltiplos alérgenos). A prevalência de alergias no mundo aumentou nas últimas décadas, e entre os fatores que contribuíram para esse aumento estão a exposição ambiental a alérgenos, as mudanças no estilo de vida, os fatores infecciosos e socioeconômicos e a grande concentração de poluentes externos ${ }^{1}$.

A alergia tem etiologia multifatorial, e a interação entre fatores genéticos e não genéticos determina a expressão da doença. Muitos estudos têm avaliado o risco de uma criança tornar-se alérgica, com base na história familiar de alergia ${ }^{2}$. As principais comorbidades associadas à rinite alérgica são: asma, conjuntivite alérgica, rinossinusite aguda e crônica, otite média com efusão, alterações bucais do desenvolvimento craniofacial em crianças que apresentam respiração oral, além de apneia e hipopneia, presentes tanto em crianças como em adultos ${ }^{3,4}$.

A maloclusão dentária pode produzir alterações nos dentes ou face, sendo considerada um problema relacionado ao crescimento e desenvolvimento dos ossos maxilares ou mandibulares durante a infância e adolescência. Esse tipo de anomalia pode ter efeitos funcionais, estéticos ou psicossociais envolvendo a mastigação, a fonação e a oclusão, com impacto negativo na vida diária dos indivíduos afetados. Sua causa se deve a interação entre fatores ambientais, congênitos, morfológicos e biomecânicos ${ }^{5,6}$. Vem sendo observada uma alta prevalência de crianças atópicas com resposta positiva ao teste alérgico, além de apresentarem padrão de respiração bucal. Alterações respiratórias possuem uma relação com deformações orofaciais, como as maloclusões, especialmente a mordida cruzada. Hábitos de respiração oronasal e de chupar chupeta evidenciaram efeitos negativos na qualidade de vida de crianças e de seus familiares ${ }^{7}$. Dessa maneira, o objetivo deste estudo foi avaliar a frequência de pacientes portadores de alergias atendidos em Clínica de Odontopediatria de instituições de ensino e a provável associação entre atopias, maloclusão, traumatismos dentoalveolares, prematuridade e hábitos bucais viciosos.

\section{MATERIAL E MÉTODO}

Este estudo obteve a aprovação do Comitê de Ética em Pesquisa da Universidade Veiga de Almeida, sob parecer número 2.369.137, e do Comitê de Ética em Pesquisa da Faculdade São Leopoldo Mandic, sob parecer número 3.404.295. Foram avaliados prontuários de todos os pacientes que procuraram atendimento odontológico nas Clínicas de Odontopediatria da Universidade Veiga de Almeida e da Faculdade São Leopoldo Mandic, unidade Rio de Janeiro, ambas instituições de ensino particulares, durante o período de julho de 2017 a agosto de 2018.

Foram coletados dados referentes ao sexo, idade, histórico médico relacionado à gestação do paciente, parto, prematuridade, histórico médico atual, presença de reação alérgica, histórico de alergias, dentição atual, presença de maloclusão (mordida aberta, mordida cruzada e apinhamento dentário), traumatismo dentário e hábitos viciosos. Todos os atendimentos foram realizados por alunos do curso de graduação em Odontologia da Universidade Veiga de Almeida e do curso de especialização em Odontopediatria da São Leopoldo Mandic sob supervisão docente.

Para análise e tratamento estatístico dos dados, foi usado o programa SPSS-21.0 por meio do método estatístico qui-quadrado, tendo como nível de significância o valor de p<0,05. 


\section{RESULTADO}

A amostra final foi composta de 136 crianças que receberam atendimento odontológico no período estudado. A faixa etária dos pacientes variou de 2 a 15 anos de idade, com uma média de 7,28 anos $( \pm 3,43)$. A maioria das crianças era do sexo masculino $(74 ; 54,4 \%)$ e saudáveis $(109 ; 80,1 \%)$.

Na Tabela 1 é observada a frequência absoluta e relativa relacionadas ao histórico médico das crianças, assim como dados relativos à gestação e ao parto. Na Tabela 2 é verificada a frequência absoluta das variáveis individuais dos participantes relacionadas à dentição. A Tabela 3 aponta a frequência absoluta e relativa referente às variáveis maloclusão, hábitos bucais viciosos, histórico de traumatismo dentário e prematuridade, em crianças e adolescentes que apresentaram ou não atopia de acordo com o relato de seus responsáveis. Foi verificada associação entre presença de atopia e histórico de traumatismos dentários, hábitos bucais viciosos e maloclusão $(p<0,05)$.

Tabela 1. Características gerais da amostra estudada $(n=136)$

\begin{tabular}{|c|c|c|c|}
\hline Variável & & Frequência absoluta (n)* & Frequência relativa (\%) \\
\hline \multirow{2}{*}{ Sexo } & Masculino & 74 & 54,4 \\
\hline & Feminino & 62 & 45,6 \\
\hline \multirow{3}{*}{$\begin{array}{l}\text { Está em } \\
\text { tratamento } \\
\text { médico }\end{array}$} & Sim & 29 & 19,9 \\
\hline & Não & 109 & 80,1 \\
\hline & Alergia, rinite, bronquite e dermatite & 17 & 12,5 \\
\hline \multirow{4}{*}{$\begin{array}{l}\text { Qual é o motivo do } \\
\text { tratamento? }\end{array}$} & Alteração no comportamento & 7 & 5,1 \\
\hline & Alteração no trato respiratório & 3 & 2,2 \\
\hline & Outros & 2 & 1,5 \\
\hline & Sim, não relatou o motivo & 6 & 4,4 \\
\hline \multirow{4}{*}{$\begin{array}{l}\text { Já apresentou } \\
\text { alguma reação } \\
\text { alérgica aguda? }\end{array}$} & Sim, por antibióticos & 2 & 1,5 \\
\hline & Sim, por outros medicamentos & 3 & 2,2 \\
\hline & Sim, por corantes & 2 & 1,5 \\
\hline & Não & 121 & 89,0 \\
\hline \multirow{4}{*}{$\begin{array}{l}\text { Seu filho tem } \\
\text { alergia? }\end{array}$} & Sim & 49 & 36,0 \\
\hline & Não & 83 & 61,0 \\
\hline & Não respondeu & 4 & 3,0 \\
\hline & Normal & 41 & 30,1 \\
\hline \multirow[t]{3}{*}{ Gestação } & Com intercorrências & 10 & 7,4 \\
\hline & Não relatou & 85 & 62,5 \\
\hline & Normal & 20 & 14,7 \\
\hline \multirow[t]{3}{*}{ Parto } & Cesárea & 27 & 19,9 \\
\hline & Dados ausentes & 89 & 65,4 \\
\hline & Sim & 9 & 6,6 \\
\hline \multirow[t]{3}{*}{ Prematuro } & Não & 113 & 83,1 \\
\hline & Dados ausentes & 14 & 10,3 \\
\hline & Sim & 6 & 4,4 \\
\hline \multirow[t]{3}{*}{ Uso da incubadora } & Não & 33 & 24,3 \\
\hline & Dados ausentes & 97 & 71,3 \\
\hline & Sim & 15 & 11,1 \\
\hline \multirow[t]{3}{*}{ Uso de mamadeira } & Não & 75 & 55,1 \\
\hline & Dados ausentes & 46 & 33,8 \\
\hline & Sim & 35 & 25,8 \\
\hline \multirow[t]{2}{*}{ Uso de chupeta } & Não & 52 & 38,2 \\
\hline & Dados ausentes & 49 & 36,0 \\
\hline
\end{tabular}

*Dados analisados por meio do teste do qui-quadrado. 
Tabela 2. Dados relativos à dentição, maloclusão, hábitos bucais viciosos e traumatismos dentários na amostra estudada $(n=136)$

\begin{tabular}{|c|c|c|c|}
\hline Variável & & Frequência absoluta (n)* & Frequência relativa (\%) \\
\hline \multirow{3}{*}{ Dentição } & Mista & 47 & 34,6 \\
\hline & Decídua & 71 & 52,2 \\
\hline & Permanente & 18 & 13,2 \\
\hline \multirow{5}{*}{ Maloclusão } & Mordida aberta anterior & 16 & 11,8 \\
\hline & Mordida aberta posterior & 8 & 5,9 \\
\hline & Outras maloclusões & 1 & 0,7 \\
\hline & Não apresenta & 60 & 44,1 \\
\hline & Dados ausentes & 51 & 37,5 \\
\hline \multirow[b]{2}{*}{ Hábitos bucais viciosos } & Sim & 87 & 64,0 \\
\hline & Não & 47 & 34,6 \\
\hline \multirow{4}{*}{$\begin{array}{c}\text { Histórico de traumatismos } \\
\text { dentários }\end{array}$} & Dados ausentes & 32 & 1,5 \\
\hline & Sim & 41 & 30,1 \\
\hline & Não & 88 & 64,7 \\
\hline & Sim, por corantes & 7 & 5,1 \\
\hline
\end{tabular}

*Dados analisados por meio do teste do qui-quadrado.

Tabela 3. Comparação das variáveis maloclusão, hábitos bucais viciosos, histórico de traumatismo dentário e prematuridade entre crianças e adolescentes atópicos e não atópicos

\begin{tabular}{|c|c|c|c|c|}
\hline Variável & & $\begin{array}{c}\text { Pacientes atópicos } \\
(n=49)\end{array}$ & $\begin{array}{c}\text { Pacientes não } \\
\text { atópicos }(n=83)\end{array}$ & $p$-valor ${ }^{*}$ \\
\hline \multirow{3}{*}{ Maloclusão n (\%) } & Sim & $6(12,24)$ & $19(22,9)$ & \multirow{3}{*}{0,044} \\
\hline & Não & $26(53,1)$ & $34(41,0)$ & \\
\hline & $\begin{array}{c}\text { Dados } \\
\text { ausentes }\end{array}$ & $17(34,7)$ & $30(36,1)$ & \\
\hline \multirow{3}{*}{ Hábitos bucais viciosos } & Sim & $32(65,3)$ & $53(63,9)$ & \multirow{3}{*}{0,003} \\
\hline & Não & $17(34,7)$ & $29(34,9)$ & \\
\hline & $\begin{array}{c}\text { Dados } \\
\text { ausentes }\end{array}$ & $\mathbf{0}$ & $1(1,2)$ & \\
\hline \multirow{2}{*}{$\begin{array}{l}\text { Histórico de traumatismos } \\
\text { dentários }\end{array}$} & Sim & $15(30,6)$ & $26(31,3)$ & \multirow[b]{2}{*}{0,002} \\
\hline & Não & $32(65,3)$ & $54(65,1)$ & \\
\hline \multirow{3}{*}{ Prematuridade } & Sim & $5(10,2)$ & $4(4,8)$ & \multirow{3}{*}{0.494} \\
\hline & Não & $40(81,6)$ & $72(86,7)$ & \\
\hline & $\begin{array}{c}\text { Dados } \\
\text { ausentes }\end{array}$ & $4(8,2)$ & $7(8,4)$ & \\
\hline \multirow[b]{3}{*}{ Uso de mamadeira } & Sim & $5(10,2)$ & $10(12,0)$ & \multirow[b]{3}{*}{0.787} \\
\hline & Não & $29(59,2)$ & $44(53,0)$ & \\
\hline & $\begin{array}{c}\text { Dados } \\
\text { ausentes }\end{array}$ & $15(30,6)$ & $29(35,0)$ & \\
\hline
\end{tabular}

*Dados analisados por meio do teste do qui-quadrado.

\section{DISCUSSÃO}

A atopia é a tendência de apresentar índices elevados de imunoglobulina E (IgE) e anticorpos para alérgenos ambientais comumente encontrados ${ }^{8}$. As manifestações clínicas mais comuns de atopia são asma, rinite alérgica e dermatite atópica ${ }^{8}$. A obstrução da via aérea nasal por causa da 
alergia atópica pode estar associada à respiração bucal e facial, favorecendo o desenvolvimento de anomalias, incluindo as maloclusões 9,10 . Neste estudo, $36 \%$ das crianças e adolescentes apresentaram alergias, segundo relato de seus responsáveis. Contudo, não foi observada nenhuma relação entre presença de atopia e manifestações clínicas e características gerais avaliadas, com exceção do histórico de traumatismo dentário, hábitos bucais viciosos e maloclusão $(p<0,05)$. 0 grupo não alérgico $(31,3 \%)$ demonstrou uma frequência ligeiramente maior de histórico de traumatismos dentários quando comparado ao grupo alérgico $(30,6 \%)$, sendo um dado significativo $(p=0,002)$. Isso pode estar relacionado a fatores de estresse psicológico e isolamento social que pacientes atópicos costumam apresentar, especialmente na exacerbação de sua condição, o que diminui seu convívio social e, consequentemente, a redução de atividades na infância que estão constantemente envolvidas na ocorrência de traumas dentários ${ }^{11-14}$.

A maioria das revisões sobre fatores de risco para asma recomenda o aleitamento estendido para reduzir a probabilidade de desenvolvimento de atopia e asma na infância ${ }^{15-17}$. Embora tal visão seja amplamente aceita e promovida, alguns estudos apresentam resultados conflitantes ${ }^{11,12}$. Alguns investigadores sugerem um risco maior de alergia e asma associado com aleitamento, enquanto outros apontam que o aleitamento apresenta função protetora. Uma das razões que explicam tal teoria seria a hipótese de que o leite produzido por mães alérgicas pode induzir a sensibilidade a alérgenos ${ }^{15}$. Essa temática é de suma importância considerando o agravo, pois se sabe que o aleitamento materno, quando comparado ao artificial, é um fator protetor contra a incidência de alergias respiratórias, especialmente a asma ${ }^{11}$. No estudo de Giuca et al. ${ }^{16}$, as crianças que receberam os benefícios do aleitamento materno apresentaram menos casos de rinite quando comparadas àquelas com aleitamento artificial exclusivo. Neste estudo, não foram investigadas informações a respeito da amamentação das crianças incluídas na amostra, entretanto foram registrados dados relativos (com baixa frequência) ao uso de mamadeiras $(11,1 \%)$ e chupetas $(25,8 \%)$, que predispõem ao aparecimento de mordidas abertas e cruzadas. Em pacientes atópicos, o uso de mamadeira apresentou uma frequência menor quando comparado ao grupo não alérgico $(10,2 \%$ e $12 \%$, respectivamente), não sendo possível observar uma associação significativa entre atopia e uso de mamadeira $(p>0,05)$.

A associação entre respiração bucal e rinite tem sido amplamente documentada e ocorre como resultado da obstrução nasal, que é um dos sintomas mais desconfortáveis da rinite ${ }^{17}$. Estudos em longo prazo mostraram uma maior frequência de desenvolvimento facial alterado e maloclusão dentária, principalmente como possível consequência da respiração bucal crônica ${ }^{18,19}$. Esses estudos estão de acordo com os achados da presente pesquisa, em que a presença de hábitos bucais viciosos, como a onicofagia e morder objetos, foi relatada em $64 \%$ dos pacientes, indicando uma alta prevalência no atual grupo estudado $(p>0,05)$. A relação entre presença de atopia e hábitos bucais viciosos demonstrou-se significativa neste estudo $(p<0,003)$, em que o grupo com alergia apresentou uma frequência de 65,3\%.

Sabe-se que a presença da mordida aberta pode dificultar o vedamento labial e favorecer a presença da respiração bucal ${ }^{20}$. Em um estudo prévio, Menezes et al. ${ }^{21}$ (2006) avaliaram indivíduos portadores de respiração bucal, e os maiores percentuais verificados relacionados a alterações faciais foram para a presença de mordida aberta anterior (60\%), selamento labial inadequado $(58,8 \%)$ e palato ogival $(38,8 \%)^{21}$. No presente estudo, foi observada uma associação entre maloclusão e atopia $(p=0,044)$, em que o grupo alérgico apresentou uma frequência menor de maloclusões quando comparado ao grupo não alérgico (12,24\% e 22,9\%, respectivamente).

A rinite alérgica é considerada uma das principais causas de obstrução das vias aéreas em crianças, o que pode induzir uma perda de respiração nasal fisiológica habitual e, consequentemente, alterar o desenvolvimento craniofacial ${ }^{22}$. Os fatores predisponentes do traumatismo dentário são a cobertura labial inadequada e o aumento do overjet, com protrusão dentária $^{22}$. Um estudo prévio com portadores de rinite alérgica demonstrou que crianças 
portadoras da patologia tinham um risco aumentado para o traumatismo dentário, concluindo haver a necessidade de uma maior atenção de profissionais da saúde e cuidadores dessas crianças $^{23}$. Diante de uma associação prévia descrita na literatura, foi feita a busca na população estudada de uma associação entre o relato de alergias e o traumatismo dentário, em que pacientes não atópicos apresentaram uma frequência maior de histórico de trauma dentário quando comparados a pacientes atópicos $(p=0,002)$.

0 parto cesariano também demonstra ser um fator importante no desenvolvimento de atopias, como dermatite atópica e alergia alimentar ${ }^{24}$. Essa associação deve-se a alterações na microbiota intestinal presentes na primeira infância desses pacientes ${ }^{24}$. Um estudo que avaliou 459 crianças que foram acompanhadas até o $36^{\circ}$ mês de idade verificou que o parto cesariano predispôs ao desenvolvimento de alergia alimentar nesses pacientes ${ }^{24}$. Além disso, crianças que possuíam pelo menos um pai alérgico apresentavam uma maior probabilidade de desenvolver alergia alimentar quando comparadas a crianças que não possuíam histórico de alergia e nasceram por meio do parto normal (OR 10,0; 95\% CI 3,06-32,7)24. No presente estudo, foi constatada uma frequência ligeiramente maior de pacientes que nasceram por parto cesariano $(19,9 \%)$ quando comparados aos que nasceram de parto normal $(14,7 \%)$, apesar de não haver nenhuma correlação entre a presença de alergia e o tipo de parto $(p>0,05)$.

A prematuridade propicia a um maior risco de desenvolvimento de asma, assim como um precoce desconforto respiratório e maior risco de infecção respiratória, quando comparada com crianças que tiveram parto normal ${ }^{25}$. Neste estudo, foi verificada uma baixa frequência de relato de prematuridade pelos responsáveis $(6,6 \%)$, não havendo associação entre atopia e prematuridade $(p>0,05)$.

Nesta pesquisa, diversos aspectos apresentaram uma alta frequência na população avaliada, como hábitos bucais viciosos (64\%) e presença de alergias (36\%). Já outros foram significativos quando correlacionados à atopia, como histórico de traumatismos dentários, maloclusão e hábitos viciosos. Contudo, alguns aspectos, como tipo de dentição, prematuridade, uso de mamadeira e chupeta, não apresentaram significância, o que pode ser justificado em razão das limitações deste estudo, como a presença de uma amostra pequena em relação ao número total de crianças atendidas anualmente e a ausência do diagnóstico médico por escrito das crianças participantes. Somente o relato dos responsáveis foi utilizado para caracterizar a presença das atopias.

\section{CONCLUSÃO}

Diante dos resultados observados neste estudo, é possível concluir que:

- 0 estudo possibilitou verificar uma alta prevalência de hábitos bucais viciosos (64\%) nas crianças atendidas na Clínica de Odontopediatria do curso de Odontologia da Universidade Veiga de Almeida.

- A prevalência de alergias relatadas nessa população foi de $36 \%$, sendo observada associação significativa entre presença de atopias e histórico de traumatismos dentários, maloclusão e hábitos bucais viciosos $(p<0,05)$.

\section{REFERÊNCIAS}

1. Leal AO, Sá GGM, Oliveira Neto JG, Carvalho DA, Monteiro MM, Martins MCC. Sinais de rinite em estudantes universitários da área da saúde. R. Interd. 2015 Jan-Mar;8(1):183-93.

2. Nogueira FLA, Calvário MAF, Fernandes JA. Reabilitação oral em paciente atópico: relato de caso. Rev Ciênc Méd Biol. 2015 Maio-Ago;14(2):246-53. http://dx.doi.org/10.9771/cmbio.v14i2.13790. 
3. Campos FL, Vazquez FL, Cortellazzi KL, Guerra LM, Ambrosano GMB, Meneghim MC, et al. A má oclusão e sua associação com variáveis socioeconômicas, hábitos e cuidados em crianças de cinco anos de idade. Rev Odontol UNESP. 2013 May-June;42(3):160-6. http://dx.doi.org/10.1590/S180725772013000300003.

4. Sakano E, Sarinho ESC, Cruz AA, Pastorino AC, Tamashiro E, Kuschnir F, et al. IV Brazilian Consensus on Rhinitis - an update on allergic rhinitis. Rev Bras Otorrinolaringol (Engl Ed). 2018 Jan;84(1):3-14. http://dx.doi.org/10.1016/j.bjorl.2017.10.006. PMid:29254864.

5. Silveira MF, Freire RS, Nepomuceno MO, Martins AM, Marcopito LF. Severity of malocclusion in adolescents: populational-based study in the north of Minas Gerais, Brazil. Rev Saude Publica. 2016;50:11. http://dx.doi.org/10.1590/S1518-8787.2016050005861. PMid:27143611.

6. Rosa GN, Buzzati BCS, Piovesan C, Mendes FM, Oliveira MDM, Ardenghi TM. Impact of malocclusion on oral health-related quality of life of preschool children. Rev Gaucha Odontol. 2015 Mar;63(1):33-40. http://dx.doi.org/10.1590/1981-863720150001000052959.

7. Carminatti M, Lavra-Pinto B, Franzon R, Rodrigues JA, Araújo FB, Gomes E. Impacto da cárie dentária, maloclusão e hábitos orais na qualidade de vida relacionada à saúde oral em crianças pré-escolares. Audiol Commun Res. 2017;22(0):e1801. http://dx.doi.org/10.1590/2317-6431-2016-1801.

8. Al Ali A, Richmond S, Popat H, Toma AM, Playle R, Pickles T, et al. A three-dimensional analysis of the effect of atopy on face shape. Eur J Orthod. 2014 Oct;36(5):506-11. http://dx.doi.org/10.1093/ejo/cjs107. PMid:25257926.

9. Izuka EN. A influência da respiração oral na oclusão dentária: uma visão geral da literatura. ACTA ORL/Técnicas em Otorrinolaringologia. 2008;26(3):151-4.

10. Branco A, Ferrari FG, Weber SAT. Alterações orofaciais em doenças alérgicas de vias aéreas. Rev Paul Pediatr. 2007 Sep;25(3):266-70. http://dx.doi.org/10.1590/S0103-05822007000300012.

11. Arima M, Shimizu Y, Sowa J, Narita T, Nishi I, Iwata N, et al. Psychosomatic analysis of atopic dermatitis using a psychological test. J Dermatol. 2005 Mar;32(3):160-8. http://dx.doi.org/10.1111/j.13468138.2005.tb00738.x. PMid:15863860.

12. Morren MA, Przybilla B, Bamelis M, Heykants B, Reynaers A, Degreef H. Atopic dermatitis: triggering factors. J Am Acad Dermatol. 1994 Sep;31(3 Pt 1):467-73. http://dx.doi.org/10.1016/S01909622(94)70213-6. PMid:8077475.

13. Granville-Garcia AF, de Menezes VA, de Lira PI. Dental trauma and associated factors in Brazilian preschoolers. Dent Traumatol. 2006 Dec;22(6):318-22. http://dx.doi.org/10.1111/j.16009657.2005.00390.x. PMid:17073924.

14. Assunção LRS, Cunha RF, Ferelle AF. Análise dos traumatismos e suas sequelas na dentição decídua: uma revisão da literatura. Pesqui Bras Odontopediatria Clin Integr. 2007 Maio-Ago;7(2):173-9. http://dx.doi.org/10.4034/1519.0501.2007.0072.0012.

15. Sorio GN, de Almeida JM. Aleitamento materno e o desenvolvimento da asma infantil. Revista Brasileira de Iniciação Científica. 2016;3(5):121-42.

16. Giuca MR, Caputo E, Nastassio S, Passini M. Correlation between otitis media and dental malocclusion in children. Eur Arch Paediatr Dent. 2011 Oct;12(5):241-4. http://dx.doi.org/10.1007/BF03262815. PMid:21993063.

17. Kim DH, Han K, Kim SW. Effects of antibiotics on the development of asthma and other allergic diseases in children and adolescents. Allergy Asthma Immunol Res. 2018 Sep;10(5):457-65. http://dx.doi.org/10.4168/aair.2018.10.5.457. PMid:30088366.

18. Barros JRC, Becker HMG, Pinto JA. Evaluation of atopy among mouth-breathing pediatric patients referred for treatment to a tertiary care center. J Pediatr (Rio J). 2006;82(6):458-64. PMid:17171205. 
19. Imbaud TC, Mallozi MC, Domingos VBTC, Solé D. Frequência de rinite e alterações orofaciais em pacientes com má oclusão dentária. Rev Paul Pediatr. 2016;34(2):184-8. http://dx.doi.org/10.1016/j.rpped.2015.10.002. PMid:26631324.

20. Zicari AM, Marzo G, Rugiano A, Celani C, Carbone MP, Tecco S, et al. Habitual snoring and atopic state: correlations with respiratory function and teeth occlusion. BMC Pediatr. 2012;12(1):175. http://dx.doi.org/10.1186/1471-2431-12-175. PMid:23134563.

21. Menezes VA, Barbosa AMF, Souza RMS, Freire CVC, Granville-Garcia AF. Ocorrência de rinite, respiração oral e alterações orofaciais em adolescentes asmáticos. Rev CEFAC. 2013 Jun;15(3):663-71. http://dx.doi.org/10.1590/S1516-18462013005000014.

22. Al-Herz W. A Systemic review on the prevalence of atopic diseases in children in the Arabian Peninsula. Med Princ Pract. 2018 Nov;27(5):436-42. http://dx.doi.org/10.1159/000493267. PMid:30149382.

23. Siao MJ, Chen GS, Lee WC, Horng JT, Chang CW, Li CH. Increased risk of dental trauma in patients with allergic rhinitis: a nationwide population-based cohort study. PLoS One. 2017;12(7):e0182370. http://dx.doi.org/10.1371/journal.pone.0182370. PMid:28759627.

24. Papathoma E, Triga M, Fouzas S, Dimitriou G. Cesarean section delivery and development of food allergy and atopic dermatitis in early childhood. Pediatr Allergy Immunol. 2016 Jun;27(4):419-24. http://dx.doi.org/10.1111/pai.12552. PMid:26888069.

25. Almqvist C, Cnattingius S, Lichtenstein P, Lundholm C. The impact of birth mode of delivery on childhood asthma and allergic diseases-a sibling study. Clin Exp Allergy. 2012 Sep;42(9):1369-76. http://dx.doi.org/10.1111/j.1365-2222.2012.04021.x. PMid:22925323.

\section{CONFLITOS DE INTERESSE}

Os autores declaram não haver conflitos de interesse.

\section{*AUTOR PARA CORRESPONDÊNCIA}

Patricia Nivoloni Tannure, UVA - Universidade Veiga de Almeida, Rua Ibituruna, 108, Maracanã, 20271-020 Rio de Janeiro - RJ, Brasil, e-mail: pntannure@gmail.com

Recebido: Setembro 27, 2020

Aprovado: Outubro 28, 2020 